\title{
B7. eVAluATION OF ANTIFUNGAL PROPERTIES OF THE CHIMERIC PROTEIN WGA-Fc AGAINST THE FUNGAL CELL WALL.
}

Susie Coutinho Liedke; Allan Jefferson Guimarães²; José Mauro Peralta².

${ }^{1}$ Universidade Federal do Rio de Janeiro;

${ }^{2}$ Universidade Federal Fluminense.

INTRODUCTION Fungal infections have emerged as a serious public health problem and most therapeutic strategies are inefficient. Therefore, it makes necessary the development of new and efficient therapeutic strategies for the treatment of mycosis. Passive immunization using monoclonal antibodies is a powerful alternative to eliminate fungal infections, mainly targeting surface structures such as chitin and glucan that are common to all fungi. However, these polysaccharides are not immunogenic and unable to induce humoral response.

OBJECTIVE In order to develop a broader reagent against mycosis, we have used lectins, dectin-1 and WGA, with recognized affinity to common fungal structures such as $\beta-1,3$-glucan and chitin, and fused to effector Fc part of IgG.

METHODOLOGY The chimeric protein WGA-Fc (IgG2a) was expressed and characterized. Initially, we showed that WGAFc chimera formed dimers in solution with quaternary structure resembling an antibody.

RESULTS Phagocytosis assays showed that the chimera has opsonizing activity in macrophages and stressed the antifungal activity of these cells in pretreated fungi with the chimera compared to controls. The protective activity was evaluated in vivo and three groups of different treatments were used: control only with PBS, native WGA and WGA-Fc chimera.C57BL/6 mice were treated in the three conditions and infected with $H$. capsulatum. One week later, the animals were sacrificed and lungs, spleen and liver were macerated and plated. The group treated with WGAFc had attenuated growth of the fungi in the lungs, spleen and liver, compared to controls. The numbers of CFU of PBS, native WGA and WGA-Fc were, respectively, $5,8 \times 10^{7}, 2,1 \times 10^{7}$ and $4,6 \times 10^{6}$ in the lungs; $3,8 \times 10^{6}, 1,6 \times 10^{6}$ and $2,4 \times 10^{5}$ in the spleen and $1,1 \times 10^{6}, 1,1 \times 10^{6}$ and $1,0 \times 10^{5}$ in the liver. Treatment with WGA-Fc followed by 
infection of mice also reduced effectively the mortality relative to control animals. PBS treated animals died by 17 days and with native WGA died by 26 days. All the animals treated with WGA-Fc chimera survived within the 60 days period of the experiment.

CONCLUSION These results showed the potential of WGA-Fc chimera as possible antifungal agent. In order to increase the spectrum of organisms, plasmids for dectin-Fc chimeras were constructed, and currently, we are expressing the proteins in CHO-k1 cells.

KEYWORDS proteína quimérica, antifúngico, micoses. 